\title{
Combination of immunotherapy and radiotherapy in the treatment of brain metastases from non-small cell lung cancer
}

\author{
Rafal Suwinski \\ Radiotherapy and Chemotherapy Clinic and Teaching Hospital, Maria Sklodowska-Curie National Research Institute of Oncology, Gliwice, Poland \\ Correspondence to: Rafal Suwinski, MD, PhD. Radiotherapy and Chemotherapy Clinic and Teaching Hospital, Maria Sklodowska-Curie National \\ Research Institute of Oncology, Gliwice Branch, 44-100 Gliwice, Wybrzeze Armii Krajowej 15, Poland. Email: rafal.suwinski@io.gliwice.pl.
}

\begin{abstract}
It was commonly assumed in the past that blood-brain barrier could efficiently prohibit penetration of large peptide molecules, such as monoclonal antibodies, including programmed death-1 (PD-1)/programmed death-ligand 1 (PD-L1) inhibitors. This belief has been recently revised by studies that demonstrate the presence of functional lymphatic vessels lining the dural sinuses. Furthermore, the activated circulating $\mathrm{T}$ cells have been shown to cross the blood-brain barrier. Such observations created strong rationale for attempts of immunotherapy for patients with brain metastases, used either alone or in combination with radiotherapy. The expected benefit from immunotherapy particularly refers to patients without targetable "driver" mutations who are not considered as candidates for novel targeted therapies. Current inference on efficacy and safety of combination of immunotherapy and radiotherapy in the treatment of brain metastases from non-small cell lung cancer (NSCLC) origins, in most, from the retrospective studies. The existing data suggest that use of immune checkpoint inhibitors (ICIs) with brain radiotherapy improves patients outcome, compared to brain radiotherapy alone. The available data also suggest that concurrent use of ICI and stereotactic radiation therapy (SRT) for brain metastases from NSCLC is tolerable and appears more effective than sequential combination of radiotherapy and ICI. Use of steroids appeared detrimental. Since a dependence between the risk of adverse events and type of ICI therapy as well as tumor pathology was found, further studies are required to establish optimal dosage, selection of drugs and sequence of ICI and brain radiotherapy in patients with brain metastases from NSCLC.
\end{abstract}

Keywords: Non-small cell lung cancer (NSCLC); brain metastases; radiotherapy; immune checkpoint inhibitor (ICI); review

Submitted Aug 06, 2020. Accepted for publication Sep 29, 2020.

doi: $10.21037 /$ jtd-2019-rbmlc-08

View this article at: http://dx.doi.org/10.21037/jtd-2019-rbmlc-08

\section{Introduction}

From the historical perspective, development of brain metastases was considered as one of the most grim and devastating events in natural course and therapy of patients with non-small cell lung cancer (NSCLC). Despite use of surgery, whole brain radiotherapy (WBRT) and best supportive care median overall survival ranged between 2 and 7 months, depending on the prognostic class of the patient (1). Recent advances in imaging, molecular biology, surgery, radiation treatment and systemic therapy considerably expanded therapeutic options available for such patients and, in most of the cases, favorably altered the prognosis. Among recent developments in systemic therapy introduction of immunotherapy for NCCLC influenced standard approach to patients with advanced disease, including those with brain metastases $(2,3)$. Combination of immunotherapy and radiotherapy (RT) became intensively explored considering the potentially beneficial interaction of these modalities. Here we provide a concise review of recent research progress on this subject.

\section{Current place of immunotherapy for NSCLC}

The first clinical approvals of immunotherapy in locally 
advanced or metastatic NSCLC referred to previously treated patients who progressed on chemotherapy (4-6).

\section{Nivolumab}

Nivolumab is a programmed death-1 (PD-1) inhibitor proven to increase overall survival (OS) over docetaxel in both squamous (4) and nonsquamous (5) NSCLC. For nonsquamous cancer the outcomes had improved with increase in programmed death-ligand 1 (PD-L1) expression, while in squamous cancer the PD-L1 expression was neither prognostic nor predictive of benefit. Two-year overall survival rates with nivolumab versus docetaxel were $23 \%$ versus $8 \%$ in squamous NSCLC and $29 \%$ versus $16 \%$ in nonsquamous NSCLC (7). In both trials $(4,5)$ patients with brain metastases were included, providing they underwent brain irradiation, had no neurological deficits and did not require high doses of steroids.

\section{Atezolizumab}

Atezolizumab the anti-PD-L1 antibody, has been approved as second-line treatment for patients with previously treated non-small-cell lung cancer based on the results of a phase II trial that demonstrated its improved efficacy compared to docetaxel (8). Patients with active or untreated CNS metastases were excluded in this trial.

Recently, major changes in systemic therapy for NSCLC have been influenced by introduction of immunotherapy to first line treatment for locally advanced or metastatic NSCLC.

\section{Durvalumab}

Durvalumab, the anti PD-L1 antibody, significantly prolonged overall survival among patients with stage III, unresectable NSCLC who did not have disease progression after concurrent chemoradiotherapy. Inclusion criteria for the pivotal PACIFIC trial $(9,10)$ precluded recruitment of patients with brain metastases.

\section{Pembrolizumab}

Pembrolizumab, PD-L1 inhibitor, was initially investigated in NSCLC PD-L1 positive patients (expression on at least $1 \%$ of tumor cells) who had two or more cycles of platinum-doublet chemotherapy (6). Those with an EGFRsensitising mutation or ALK gene rearrangement were eligible, providing they progressed on appropriate tyrosine kinase inhibitor therapy. Patients with known active brain metastases were excluded. Further studies of pembrolizumab referred to first line of treatment for patients with metastatic NSCLC. It was approved as a single agent for the first-line treatment of patients with metastatic NSCLC whose tumors express PD-L1 (Tumor Proportion Score TPS $\geq 50 \%$ ) (11). Recently, based on results of the KEYNOTE-042 trial (12), it's use in monotherapy has been expanded to include the first-line treatment of patients with stage III NSCLC who are not candidates for surgical resection or definitive chemoradiation and for patients with metastatic NSCLC with no EGFR or ALK genomic aberrations and TPS $\geq 1 \%$. Pembrolizumab has been also shown to significantly improve overall survival in combination with pemetrexed and platinum chemotherapy, as first-line treatment of patients with metastatic nonsquamous NSCLC, with no EGFR or ALK genomic tumor aberrations (13). Another pivotal trial shown improved survival for pembrolizumab in combination with carboplatin and paclitaxel as first-line treatment of patients with metastatic squamous cell lung cancer (14).

In general, patients with untreated brain metastases were not included in trials that investigated the safety and activity of pembrolizumab in first line treatment for patients with metastatic NSCLC, nevertheless, patients after brain RT or surgery could be included. For example, $11.7 \%$ of the patients from KEYNOTE-024 trial on pembrolizumab as a single agent for the first-line treatment of patients with metastatic NSCLC had brain metastases (11). Subgroup analysis from this trial suggest that the benefit, in terms of progression-free survival, from pembrolizumab was comparable in patients with brain metastases and no brain metastases with hazard ratios of 0.50 and 0.55 respectively.

\section{Radiotherapy and brain metastases from NSCLC}

When assessing the efficacy and tolerance of combined RT and immunotherapy for brain metastases it is necessary to consider the diversity of radiation treatment techniques and dose fractionation prescription practices that are employed in such treatment. Historically, the management of brain metastases with RT was performed using WBRT. The advances in tumor imaging and in conformal delivery of radiation shifted clinical interest toward stereotactic radiation therapy (SRT), particularly in patients with small number of brain metastases. Initial trials suggested clinical benefit from combining WBRT and SRT, mostly 
in terms of reduction of recurrences in the brain $(15,16)$. A concern appeared, however, regarding higher incidence of neurocognitive functional deficits in WBRT + SBRT, compared to SRT alone (17). Therefore, despite trials showing lower rates of intracranial failures with WBRT + SRT, compared to SRT alone, an increase in neurocognitive deficits, lack of survival improvement as well as new clinical data on effectiveness and tolerance of treatment prevail in favor of SRT alone, whenever such treatment is feasible (18). The quality of life is considered, thus, equally, or even more important than control rate in the brain. Regardless of preferences one has to recognize the abovementioned differences in treatment tolerance and effectiveness according to RT technique and fractionation when assessing the tolerance of combined ICI and RT.

\section{Targeted therapy vs. immunotherapy for brain metastases from NSCLC}

When deciding upon treatment selection for patients with brain metastases from NSCLC it is necessary to appreciate recent advances in treatment for tumors with targetable "driver" mutations. High effectiveness of new generation epidermal growth factor (EGFR) receptor tyrosine kinase inhibitors, such as osimertinib, in patients with EGFR mutation and brain metastases (19) or alectinib in ALK (anaplastic lymphoma kinase) mutation-positive NSCLC patients with brain metastases (20) advocate in favor of targeted therapy as first line treatment for tumors with such genetic alterations. Importantly, thus, most of the trials on immunotherapy for patients with NSCLC excluded the patients with targetable "driver" mutations, including those with brain metastases.

\section{Immunotherapy alone for brain metastases from NSCLC}

It was commonly assumed in the past that blood-brain barrier could efficiently prohibit penetration of large peptide molecules, such as monoclonal antibodies, including PD-1/ PD-L1 inhibitors. Furthermore, it was believed that central nervous system had no classical lymphatic drainage. This belief has been recently revised by studies that demonstrate the presence of functional lymphatic vessels lining the dural sinuses (21). Instead of an immune privileged location, the brain is currently considered as actively regulated site of immune surveillance (22). It is, however, also recognized that endothelial, epithelial and glial brain barriers establish compartments that differ considerably with regard to their accessibility to immune-cell subsets (23).

While CNS penetration of PD-1/PD-L1 inhibitors has not been extensively studied, the activated circulating $\mathrm{T}$ cells have been shown to cross the blood-brain barrier (23). This created strong rationale to study the effect of immunotherapy in patients with untreated brain metastases. Goldberg et al. (24) prospectively assessed 36 patients (18 with melanoma and 18 with NSCLC) with untreated or progressive brain metastases. Four of 18 patients with melanoma (22\%) and 6 of 18 patients with NSCLC $(33 \%)$ responded to treatment. This included four durable responses among patients with NSCLC that lasted over 6 months. The safety profile was considered acceptable. This study demonstrated, thus, activity of pembrolizumab in untreated or progressive brain metastases in patients with NSCLC and melanoma. Similar results were obtained in the studies investigating the activity of nivolumab in patients with untreated or progressing brain metastases (25), including both patients with squamous (26) and nonsquamous (27) lung cancer. Median overall survival reported in these studies $(26,27)$ was 5.8 and 8.6 months for squamous and non-squamous cancer respectively.

The exploratory analysis of the phase III OAK study that compared atezolizumab and docetaxel in patients with previously treated non-small-cell lung cancer focused on patients with asymptomatic, treated brain metastases (28). Median overall survival in this subset of the patients tended to be longer with atezolizumab compared to docetaxel (16.0 vs. 11.9 months), although the difference was not statistically significant (HR 0.74; 95\% CI: 0.49-1.13).

In general, thus, the studies presented here, while demonstrating the activity of immunotherapy alone for brain metastases suggest, also, the necessity to combine this treatment with other active therapies to improve still relatively poor prognosis.

\section{Sequencing of immunotherapy and radiotherapy in the treatment of brain metastases from NSCLC}

Several recent studies document synergy between extracranial radiation therapy and immune checkpoint inhibitors (ICIs). Durvalumab, approved as consolidation therapy after concurrent chemoradiation, is among the most spectacular examples $(9,10)$. A secondary analysis of KEYNOTE-001 trial of phase I pembrolizumab in advanced NSCLC also suggested synergy between 
Table 1 The original studies containing the data on brain radiotherapy combined with immune checkpoint inhibitors (ICI) in non-small cell lung cancer patients with brain metastases

\begin{tabular}{lccc}
\hline Study & ICl & No of patients & Sequence of ICl and RT \\
\hline Patruni et al. (30) & Diverse & 545 & N/A \\
Hendriks et al. (31) & PD-1/PD-L1 inhibitors & $173 / 1,025^{\star}$ & sequential: RT before ICl \\
Chen et al. (32) & PD-1 inhibitors & $157 / 260^{\star}$ & sequential vs. concurrent \\
Kotecha et al. (33) & PD-1/PD-L1 inhibitors & $99 / 150^{\star}$ & sequential vs. concurrent \\
Koenig et al. (34) & PD-1/PD-L1 inhibitors & $45 / 97^{*}$ & sequential vs. concurrent \\
Singh et al. (35) & PD-1 inhibitors & $39 / 85^{\star}$ & concurrent \\
Schapira et al. (36) & PD-1/PD-L1 inhibitors & 37 & sequential vs. concurrent \\
Ahmed et al. (37) & PD-1/PD-L1 inhibitors & 17 & sequential vs. concurrent \\
Lin et al. (38) & Atezolizumab & 1 & concurrent \\
\hline
\end{tabular}

*, the proportion of patients with brain metastases from non-small cell lung cancer (NSCLC) among all patients included in the study.

extracranial RT and immunotherapy; previous treatment with RT in patients with advanced NSCLC resulted in longer progression-free survival and overall survival with pembrolizumab than that observed in patients who did not have previous RT (29).

Following encouraging reports on successful and safe combination of ICIs and extracranial RT one may identify new studies focused on safety and effectiveness combination of ICIs and WBRT or SRT for brain metastases from NSCLC (30-38). Table 1 summarizes these reports and directs to the respective references.

The study based on the largest number of patients, published, however, only in abstract (30), compares the outcomes in patients with brain metastases from NSCLC receiving intracranial RT with or without immunotherapy. The survival data of 545 patients who received immunotherapy and of 13,998 patients who did not receive immunotherapy were extracted from the National Cancer Database. Unfortunately, no detail information on immunotherapy used or sequencing of RT and immunotherapy was provided. Use of immunotherapy significantly improved survival (median 13.1 vs. 9.7 months), and the significance was maintained in propensity score matched comparison. While this analysis is prompt to biases typical for the retrospective studies it provides strong support for efficacy of immunotherapy combined with intracranial RT in patients with brain metastases from NSCLC.

Hendriks et al. (31) compared the outcome of patients with NSCLC and brain metastases or no brain metastases treated with ICI. Out of 1,025 patients included in the study 173 were treated with brain RT before ICI (72 with WBRT, 99 with SRT and 2 with WBRT+SBRT). The response rates of patients with brain metastases or without brain metastases were similar (20.6\% vs. $22.7 \%$ ), median OS was 8.6 vs. 11.4 months respectively. When adjusted for cofounding factors the presence of brain metastases appeared not associated with poorer survival. Corticosteroids use appeared to negatively influence survival.

Seven, out of 9 studies presented in Table 1 were focused on sequencing of brain RT and ICI (31-34,36,37). Chen et al. (32) compared the outcome of NSCLC patients with brain metastases treated with SRT alone (SRT, $n=181$ ), sequential SRT and ICI $(\mathrm{n}=51)$ and concurrent SRT and ICI $(n=28)$. Median overall survival was $12.9,14.5$ and 24.7 months, respectively, and the differences were statistically significant, in favor of concurrent SRT + ICI, also when adjusted for confounding factors. Importantly, no significant differences in acute neurologic toxicity were found. Also, no significant differences with respect to immune related adverse events were detected among the patients who received sequential $v s$. concurrent SRT+ICI.

Kotecha et al. (33) reported on impact of sequencing of PD-1/PD-L1 inhibitors and SRT for patients with brain metastases from tumors at various primary sites. Out of 150 patients included in the study 99 (66\%) had brain metastases from NSCLC. Concurrent use of SRT and ICI was associated with improved response rate and duration of response in this study. Use of steroids appeared detrimental.

The detrimental effect of steroids in combined brain 
radiotherapy and immunotherapy can be explained by well-known immunosuppressant effect of these drugs. Clearly, use of steroids may diminish the beneficial immunostimulant effect of ICI. Furthermore, patients with neurological symptoms from brain metastases (and hence with worse prognosis) may more often be treated with steroids, which further enhance the observed detriment from steroids in non-randomized studies. Replacement of steroids by antioedematous drugs such as mannitol or hypertonic saline may be considered in combined brain radiotherapy and immunotherapy, whenever clinically feasible. Steroids, nevertheless, have well-established and dominant role in control of the inflammatory immunerelated adverse events.

Koenig et al. (34) analyzed disease control and adverse effects of SRT and ICI in 97 patients with brain metastases from various primary sites, including 45 patients (46\%) with NSCLC. Concurrent use of SRT and ICI improved overall survival and intracranial disease control compared to sequential use of SRT and ICI. Concurrent SRT-ICI was considered tolerable, although the incidence of radiation adverse events was higher in concurrent SRT-ICI, compared to sequential treatment ( $8 \%$ vs. $2 \%)$. Two other studies compared sequential $v s$. concurrent SRT + ICI in patients with brain metastases from NSCLC, both demonstrating favorable outcome of patients treated concurrently $(36,37)$.

Singh et al. (35) reported on concurrent use of SRT and ICI in 39 PD-L1 positive patients with NSCLC. Concurrent SRT+ICI resulted in a faster and better volumetric response in large lesions, compared to chemotherapy with SRT.

While no randomized studies comparing concurrent $v$ s. sequential SRT and ICI in patients with brain metastases from NSCLC were identified, the above evidence from the retrospective studies strongly advocates in favor of concurrent treatment.

An interesting case report (38) demonstrated extracranial abscopal effect induced by brain radiotherapy in patient treated with lung adenocarcinoma who had received atezolizumab, that was later combined with brain radiation for brain metastasis. Abscopal effects are relatively frequently in patients with malignant melanoma treated with combined radiotherapy and immunotherapy. A report by Lin et al. is, however, perhaps the first to demonstrate the ability of radiotherapy for brain metastases to induce tumor response at sites that are far beyond the irradiated volume.

In addition to the original studies on combination of radiotherapy and immunotherapy in patients with brain metastases from NSCLC a valuable insight one may find in recent review papers that focus on this subject $(2,3,39)$. Some additional information one may also find in metaanalysis of individual patient data that focused on SRT plus ICI treatment of brain metastases from various primary sites (40), with NSCLC representing only minority of the included studies. Also, a review paper on combination of radiotherapy and immunotherapy in patients with brain metastases from various primary includes some relevant data on patients with NSCLC (41).

\section{Safety of concurrent SRT and immunotherapy}

Concurrent use of two treatment modalities should always raise cautiousness about possible additive or supra-additive toxicity of such approach. Hubbeling et al. (42) reported on 163 patients with brain metastases from NSCLC treated with SRT $(\mathrm{n}=94)$, WBRT $(\mathrm{n}=101)$ and partial brain RT $(\mathrm{n}=28)$. Fifty patients received ICI in combination with RT. Treatment with an ICI and cranial RT was not associated with a significant increase in RT-related adverse events. Furthermore, the sequence and timing of PD-1 pathway inhibitor administration with respect to RT did not affect RT-related toxicity in this study. This findings are in good accordance with outcomes of studies by Chen et al. (32) and Ahmed et al. (37) who did not find increase in toxicity among diverse sequencing of treatment.

By contrast, Martin et al. (43) found symptomatic necrosis in 23/ 115 (20.0\%) vs. 25/365 (6.8\%) of patients with brain metastases from various primary sites, who did or did not receive immunotherapy, respectively. Risk of necrosis was, however, strongly associated with use of ipilimumab for melanoma. Considering clear dependence of adverse events on ICI type and tumor pathology the applicability of these data to patients with NSCLC is limited. Such censure also refers to the study by Koenig et al. (34) who found that the incidence of radiation adverse events was higher in concurrent SRT-ICI, compared to sequential treatment ( $8 \%$ vs. $2 \%$ ). Notably, however, this study recruited the patients with various primary sites, including $46 \%$ with melanoma.

While the existing retrospective studies support concurrent use of ICI and SRT for patients with brain metastases from NSCL the ongoing prospective trials, that are reviewed by other authors (3) will more precisely address this important issue. 


\section{Acknowledgments}

Funding: None.

\section{Footnote}

Provenance and Peer Review: This article was commissioned by the Guest Editor (Lucyna Kepka) for the series "Radiotherapy for Brain Metastases from Lung Cancer" published in Fournal of Thoracic Disease. The article has undergone external peer review.

Conflicts of Interest: The author has completed the ICMJE uniform disclosure form, available at http://dx.doi. org/10.21037/jtd-2019-rbmlc-08. The series "Radiotherapy for Brain Metastases from Lung Cancer" was commissioned by the editorial office without any funding or sponsorship. The author reports grants and other from Bristol-Myers-Squibb, grants and other from Merck Sharp \& Dohme, grants and other from Astellas Pharma, outside the submitted work.

Ethical Statement: The author is accountable for all aspects of the work in ensuring that questions related to the accuracy or integrity of any part of the work are appropriately investigated and resolved.

Open Access Statement: This is an Open Access article distributed in accordance with the Creative Commons Attribution-NonCommercial-NoDerivs 4.0 International License (CC BY-NC-ND 4.0), which permits the noncommercial replication and distribution of the article with the strict proviso that no changes or edits are made and the original work is properly cited (including links to both the formal publication through the relevant DOI and the license). See: https://creativecommons.org/licenses/by-nc-nd/4.0/.

\section{References}

1. Gaspar L, Scott C, Rotman M, et al. Recursive partitioning analysis (RPA) of prognostic factors in three Radiation Therapy Oncology Group (RTOG) brain metastases trials. Int J Radiat Oncol Biol Phys 1997;37:745-51.

2. Protopapa M, Kouloulias V, Nikoloudi S, et al. From Whole-Brain Radiotherapy to Immunotherapy: A Multidisciplinary Approach for Patients with Brain Metastases from NSCLC. J Oncol 2019;2019:3267409.

3. El Rassy E, Botticella A, Kattan J, et al. Non-small cell lung cancer brain metastases and the immune system:
From brain metastases development to treatment. Cancer Treat Rev 2018;68:69-79.

4. Brahmer J, Reckamp KL, Baas P, et al. Nivolumab versus Docetaxel in Advanced Squamous-Cell Non-Small-Cell Lung Cancer. N Engl J Med 2015;373:123-35.

5. Borghaei H, Paz-Ares L, Horn L, et al. Nivolumab versus Docetaxel in Advanced Nonsquamous Non-Small-Cell Lung Cancer. N Engl J Med 2015;373:1627-39.

6. Herbst RS, Baas P, Kim DW,et al. Pembrolizumab versus docetaxel for previously treated, PD-L1-positive, advanced non-small-cell lung cancer (KEYNOTE-010): a randomized controlled trial. Lancet 2016;387:1540-50.

7. Horn L, Spigel DR, Vokes EE, et al. Nivolumab Versus Docetaxel in Previously Treated Patients With Advanced Non-Small-Cell Lung Cancer: Two-Year Outcomes From Two Randomized, Open-Label, Phase III Trials (CheckMate 017 and CheckMate 057). J Clin Oncol 2017;35:3924-33

8. Fehrenbacher L, Spira A, Ballinger M, et al. Atezolizumab versus docetaxel for patients with previously treated non-small-cell lung cancer (POPLAR): a multicentre, open-label, phase 2 randomised controlled trial. Lancet 2016;387:1837-46.

9. Antonia SJ, Villegas A, Daniel D, et al. Durvalumab after Chemoradiotherapy in Stage III Non-Small-Cell Lung Cancer. N Engl J Med 2017;377:1919-29.

10. Antonia SJ, Villegas A, Daniel D, et al. Overall Survival with Durvalumab after Chemoradiotherapy in Stage III NSCLC. N Engl J Med 2018;379:2342-50.

11. Reck M, Rodríguez-Abreu D, Robinson AG, et al. Pembrolizumab versus Chemotherapy for PD-L1Positive Non-Small-Cell Lung Cancer. N Engl J Med 2016;375:1823-33.

12. Mok TSK, Wu YL, Kudaba I, et al. Pembrolizumab versus chemotherapy for previously untreated, PD-L1expressing, locally advanced or metastatic non-small-cell lung cancer (KEYNOTE-042): a randomised, open-label, controlled, phase 3 trial. Lancet 2019;393:1819-30.

13. Gandhi L, Rodríguez-Abreu D, Gadgeel S, et al. Pembrolizumab plus Chemotherapy in Metastatic NonSmall-Cell Lung Cancer. N Engl J Med 2018;378:2078-92.

14. Paz-Ares L, Luft A, Vicente D, et al. Pembrolizumab plus Chemotherapy for Squamous Non-Small-Cell Lung Cancer. N Engl J Med 2018;379:2040-51.

15. Andrews DW, Scott CB, Sperduto PW, et al. Whole brain radiation therapy with or without stereotactic radiosurgery boost for patients with one to three brain metastases: phase III results of the RTOG 9508 randomised trial. Lancet 
2004;363:1665-72.

16. AoyamaH, Shirato H, Tago M, et al. Stereotactic Radiosurgery Plus Whole-Brain Radiation Therapy vs. Stereotactic Radiosurgery Alone for Treatment of Brain Metastases A Randomized Controlled Trial. JAMA 2006;295:2483-91.

17. Chang EL, Wefel JS, Hess KR, et al. Neurocognition in patients with brain metastases treated with radiosurgery or radiosurgery plus whole-brain irradiation: a randomised controlled trial. Lancet Oncol 2009;10:1037-44.

18. Kayama T, Sato S, Sakurada K, et al. Effects of Surgery With Salvage Stereotactic Radiosurgery Versus Surgery With Whole-Brain Radiation Therapy in Patients With One to Four Brain Metastases (JCOG0504): A Phase III, Noninferiority, Randomized Controlled Trial. J Clin Oncol 2018 [Epub ahead of print].

19. Reungwetwattana T, Nakagawa K, Cho BC, et al. CNS Response to Osimertinib Versus Standard Epidermal Growth Factor Receptor Tyrosine Kinase Inhibitors in Patients With Untreated EGFR-Mutated Advanced NonSmall-Cell Lung Cancer. J Clin Oncol 2018. [Epub ahead of print]. doi: 10.1200/JCO.2018.78.3118.

20. Gadgeel S, Peters S, Mok T, et al. Alectinib versus crizotinib in treatment-naive anaplastic lymphoma kinase-positive (ALK+) non-small-cell lung cancer: CNS efficacy results from the ALEX study. Ann Oncol 2018;29:2214-22.

21. Louveau A, Smirnov I, Keyes TJ, et al. Structural and functional features of central nervous system lymphatic vessels. Nature 2015;523:337-41.

22. Negi N, Das BK. CNS: Not an immunoprivilaged site anymore but a virtual secondary lymphoid organ. Int Rev Immunol 2018;37:57-68.

23. Engelhardt B, Vajkoczy P, Weller RO. The movers and shapers in immune privilege of the CNS. Nat Immunol 2017;18:123-31.

24. Goldberg SB, Gettinger SN, Mahajan A, et al.

Pembrolizumab for patients with melanoma or non-smallcell lung cancer and untreated brain metastases: early analysis of a non-randomised, open-label, phase 2 trial. Lancet Oncol 2016;17:976-83.

25. Dudnik E, Yust-Katz S, Nechushtan H, et al. Intracranial response to nivolumab in NSCLC patients with untreated or progressing CNS metastases. Lung Cancer 2016;98:114-7.

26. Cortinovis D, Chiari R, Catino A, et al. Italian Cohort of the Nivolumab EAP in Squamous NSCLC: Efficacy and Safety in Patients With CNS Metastases. Anticancer Res 2019;39:4265-71.
27. Crinò L, Bronte G, Bidoli P, et al. Nivolumab and brain metastases in patients with advanced non-squamous nonsmall cell lung cancer. Lung Cancer 2019;129:35-40.

28. Gadgeel SM, Lukas RV, Goldschmidt J, et al. Atezolizumab in patients with advanced non-small cell lung cancer and history of asymptomatic, treated brain metastases: Exploratory analyses of the phase III OAK study. Lung Cancer 2019;128:105-12.

29. Shaverdian N, Lisberg AE, Bornazyan K, et al. Previous radiotherapy and the clinical activity and toxicity of pembrolizumab in the treatment of non-small-cell lung cancer: a secondary analysis of the KEYNOTE-001 phase 1 trial. Lancet Oncol 2017;18:895-903.

30. Patruni S, Khattab A, Abel S, et al. A comparative analysis of survival in patients with non-small cell lung cancer with brain metastases receiving intracranial radiation with and without immunotherapy. J Clin Oncol 2019;37:9025.

31. Hendriks LE, Henon C, Auclin E, et al. Outcome of patients with non-small cell lung cancer and brain metastases treated with checkpoint inhibitors. J Thorac Oncol 2019; 14:1244-54.

32. Chen L, Douglass J, Kleinberg L, et al. Concurrent Immune Checkpoint Inhibitors and Stereotactic Radiosurgery for Brain Metastases in Non-Small Cell Lung Cancer, Melanoma, and Renal Cell Carcinoma. Int J Radiat Oncol Biol Phys 2018;100:916-25.

33. Kotecha R, Kim JM, Miller JA, et al. The Impact of Sequencing PD-1/PD-L1 Inhibitrs and Stereotactic Radiosurgery for Patients with Brain Metastasis. Neuro Oncol 2019;21:1060-8.

34. Koenig JL, Shi S, Sborov K, et al. Adverse radiation effect and disease control in patients undergoing stereotactic radiosurgery and immune checkpoint inhibitor therapy for brain metastases. World Neurosurg 2019;126:e1399-411.

35. Singh C, Quian J, Yu J, et al. Local tumor response and survival outcomes after combined stereotactic radiosurgery and immunotherapy in non-small cell lung cancer with brain metastases. J Neurosurg 2019;132:512-7.

36. Schapira E, Hubbeling H, Yeap B, et al. Improved overall survival and locoregional disease control with concurrent PD-1 pathway inhibitors and stereotactic radiosurgery for lung cancer patients with brain metastases. Int J Radiat Oncol Biol Phys 2018;101:624-9.

37. Ahmed KA, Kim S, Arrington J, et al. Outcomes targeting the PD-1/PD-L1 axis in conjunction with stereotactic radiation for patients with non-small cell lung cancer brain metastases. J Neurooncol 2017;133:331-8.

38. Lin X, Lu T, Xie Z, et al. Extracranial abscopal effect 
induced by combining immunotherapy with brain radiotherapy in a patient with lung adenocarcinoma: A case report and literature review. Thoracic Cancer 2019;10:1272-5.

39. Niu J, Zhou J, Lindebak S., et al. Systemic therapy in patients with NSCLC with brain metastasis: the emerging role of immunotherapy. Neuroimmunol Neuroinflammation 2018;5:32.

40. Lehrer EJ, Peterson J, Brown PD, et al. Treatment of brain metastases with stereotactic radiosurgery and immune checkpoint inhibitors: An international meta-analysis of individual patient data. Radiother Oncol 2019;130:104-12.
41. Trapani S, Manicone M, Sikokis A Effectiveness and safety of "real" concurrent stereotactic radiotherapy and immunotherapy in metastatic solid tumors: a systematic review. Crit Rev Oncol Hematol 2019;142:9-15.

42. Hubbeling HG, Schapira EF, Horick NK, et al. Safety of Combined PD-1 Pathway Inhibition and Intracranial Radiation Therapy in Non-Small Cell Lung Cancer. J Thorac Oncol 2018;13:550-8.

43. Martin AM, Cagney DN, Catalano PJ, et al. Immunotherapy and symptomatic radiation necrosis in patients with brain metastases treated with stereotactic radiation. JAMA Oncol 2018;4:1123-4.

Cite this article as: Suwinski R. Combination of immunotherapy and radiotherapy in the treatment of brain metastases from non-small cell lung cancer. J Thorac Dis 2021;13(5):3315-3322. doi: 10.21037/jtd-2019-rbmlc-08 